\title{
Empfehlungen zur Diagnostik der exogen-allergischen Alveolitis
} Arbeitsgemeinschaft Exogen-Allergische Alveolitis der Deutschen Gesellschaft für Pneumologie und Beatmungsmedizin e. V. (DGP) und der Deutschen Gesellschaft für Allergologie und Klinische Immunologie (DGAKI)

\author{
Guidelines for Diagnosing Extrinsic Allergic Alveolitis (Hypersensitivity Pneumonitis) \\ (German Extrinsic Allergic Alveolitis Study Group)
}

Autor

Institute
J. Sennekamp ${ }^{1}$, D. Müller-Wening ${ }^{2}$, M. Amthor ${ }^{3}$, X. Baur ${ }^{4}$, K.-Ch. Bergmann $^{5}$, U. Costabel ${ }^{6}$, D. Kirsten ${ }^{7}$, D. Koschel ${ }^{8}$, R. Kroidl ${ }^{9}$, G. Liebetrau ${ }^{10}$, D. Nowak ${ }^{11}$, J. Schreiber ${ }^{12}$, C. Vogelmeier ${ }^{13}$

Die Institutsangaben sind am Ende des Beitrags gelistet. eingereicht 5.9 .2006

akzeptiert 7.9 .2006

Bibliografie

Dol $10.1055 / \mathrm{s}-2006-944326$ Pneumologie 2007; 61; 52-56 (c) Georg Thieme Verlag KG Stuttgart $\cdot$ New York ISSN 0934-8387

Korrespondenzadressen Prof. Dr. med. J. Sennekamp Labor und Praxis Prof. Sennekamp Weberstr. 118 53113 Bonn sennekamp@t-online.de Dr. D. Müller-Wening Zusamklinik - Deutsche Rentenversicherung Schwaben Paracelsusstr. 3 86441 Zusmarshausen d.mueller-wening@ drv-schwaben.de
Diese Empfehlungen aktualisieren frühere deutsche Empfehlungen [6], insbesondere die Empfehlungen dieser Arbeitsgemeinschaft aus dem Jahre 1990 unter der Federführung von K-.Ch. Bergmann [3] und berücksichtigen internationale Leitlinien $[31,35,39,45]$. Neue Erkenntnisse durch Anwendung der Computertomographie $[8,15,24,46]$, zur Wertigkeit der Symptome [12,20,26], zur chronischen Verlaufsform und Histologie derallergischen Alveolitis [2,7,30,39] sowie neue Krankheitsbilder [12,17,18, 28,36-38,43] machen diese Aktualisierung erforderlich. Diese Empfehlungen gelten nur für die inhalativ erworbene exogen-allergische Alveolitis.

Mit dem Begriff „Exogen-allergische Alveolitis (EAA)“ wird eine Gruppe von seltenen Lungenerkrankungen bezeichnet, die charakterisiert ist durch eine allergisch bedingte Entzündungsreaktion der Alveolarwand und des Interstitiums mehrere Stunden nach der Inhalation von Antigenen. Die Antigene (Partikelgröße $<5 \mu \mathrm{m}$ ) sind in Stäuben, z. B. Vogelstaub, Heustaub, Holzstaub oder in Aerosolen, Dämpfen und Gasen enthalten.

Über 60 verschiedene Antigene, hoch- oder niedrigmolekular (Haptene), unterschiedliche Expositionsintensitäten und -intervalle sowie akute und chronische Krankheitsstadien sind Faktoren, die zu sehr unterschiedlich ausgeprägten Symptomen führen. Die Diagnostik und Begutachtung ist daher in Einzelfällen schwierig, für den Erkrankten aber von erheblicher Bedeutung, da eine frühe Diagnosestellung Spätschäden verhindern kann. Die nachfolgende Empfehlung zur rationellen Diagnostik soll helfen, die Diagnosestellung der EAA zu erleichtern.

Bei der EAA ist eine Fülle diagnostischer Parameter bekannt. Diese werden zunächst besprochen, bevor auf deren Wertigkeit eingegangen und ein einfacher Weg zur zuverlässigen Diagnostik aufgezeigt wird.

\section{Klinische Symptome und Befunde}

Nach einer Latenzzeit von 3-12 Stunden nach Antigenkontakt finden sich die Leitsymptome Grippesymptomatik, Belastungsdyspnoe und Husten. Über 95\% der Patienten weisen eines oder mehrere dieser 3 Symptome auf. Alle wichtigen Symptome sind in Tab. 1 aufgeführt. Diagnostisch besonders aussagekräftig sind die Symptome, wenn sie jeweils 3-12 Stunden nach Antigenkontakt auftreten oder sich verstärken. Bei einer primär chronischen Verlaufsform steht die Belastungsdyspnoe im Vordergrund und Grippesymptome fehlen meist vollständig.

Ein differenzialdiagnostisch besonders wichtiger Auskultationsbefund ist das endinspiratorische Knisterrasseln, die so genannte Sklerophonie, die z.B. bei der toxischen Alveolitis, insbesondere dem „Organic Dust Toxic Syndrome“ (ODTS) nicht beobachtet wird [29].

Die akute Verlaufsform folgt meist einer kurzzeitigen starken Antigenexposition, beginnt 3-12 Stunden nach Antigenkontakt und kann bis maximal 48 Stunden nach Antigenkontakt anhalten. Die subakute Verlaufsform ist durch eine rezidivierende, meist geringere Allergenexposition bedingt und kann mehrere Monate andauern. Die chronische Verlaufsform mit einer Symptomdauer von einigen Monaten bis mehreren Jahren ist Folge einer persistierenden niedrigen Antigenexposition oder kann sich aus einer subakuten Verlaufsform entwickeln $[25,31,41]$.

\section{Antigenexposition}

In der Regel ist der Patient inhalativ gegenüber Stäuben, z.B. Vogelstaub, Heustaub, Holzstaub oder Aerosolen, Dämpfen oder Gasen exponiert gewesen. Darin ursächliche Antigene können Bakterien, Schimmel- oder Hefepilze, Parasiten, 
Tab. 1 Die häufigsten klinischen Symptome und Befunde der EAA [21,43]

\begin{tabular}{|l|c|}
\hline Grippesymptomatik & $91 \%$ \\
\hline Dyspnoe & $85 \%$ \\
\hline Husten & $82 \%$ \\
\hline Sklerophonie (Knisterrasseln) & $73 \%$ \\
\hline Fieber & $67 \%$ \\
\hline Schüttelfrost, Frösteln & $56 \%$ \\
\hline Auswurf & $51 \%$ \\
\hline Müdigkeit, Abgeschlagenheit & $47 \%$ \\
\hline Thoraxengegefühl & $42 \%$ \\
\hline Gewichtsverlust & $31 \%$ \\
\hline Schwitzen & $29 \%$ \\
\hline Kopfschmerzen & $25 \%$ \\
\hline Trommelschlegelfinger, Uhrglasnägel & $21 \%$ \\
\hline Übelkeit (Nausea) & $19 \%$ \\
\hline Inappetenz & $18 \%$ \\
\hline Rhinitis, Pharyngitis & $15 \%$ \\
\hline Muskelschmerzen & $14 \%$ \\
\hline Schwindel & $12 \%$ \\
\hline Hämoptyse & $8 \%$ \\
\hline
\end{tabular}

tierische oder pflanzliche Eiweiße, organische oder anorganische Chemikalien sein.

Medikamentös induzierte allergische Alveolitiden stellen eine besondere Form der exogen-allergischen Alveolitis dar, für die diese Diagnose-Empfehlungen nicht gelten. Das ursächliche Antigen gelangt hier in der Regel hämatogen in die Lunge; es sind keine Antikörper im Serum nachweisbar. Der Lymphozytentransformationstest kann hier von Bedeutung sein.

Da die allergische Alveolitis eine Berufskrankheit sein kann, ist die Unterscheidung zwischen Antigenen am Arbeitsplatz und im privaten Bereich von Bedeutung.

Die umfangreiche und gezielte Anamnese des Patienten (Berufsanamnese, Freizeitanamnese, Wohngegebenheiten, Tierkontakte, Medikamentenanamnese) ist Voraussetzung zur Expositionsermittlung. Gegebenenfalls können eine Besichtigung der Arbeitsstelle bzw. des häuslichen Umfeldes und/oder der kulturelle Nachweis (z.B. von Schimmelpilzen) sowie die Messung von Substanzen in der Luft (Isozyanate, Schimmelpilzsporen) zur Feststellung der Antigenexposition beitragen. Die häufigsten Antigene und Krankheitsbilder sind in $\bullet$ Tab. 2 aufgeführt [43].

\section{Labor}

Die häufigsten pathologischen Laborbefunde der EAA sind: Leukozytose (76\% der Fälle), erhöhtes CRP (72\%), erhöhtes SerumImmunglobulin G $(65 \%)$ und beschleunigte Blutsenkung (46\%) [43].

\section{Antigenspezifische Sensibilisierung}

Die Sensibilisierung des Patienten wird durch spezifische Antikörper vom IgG-Typ im Serum nachgewiesen. Bei manchen Antigenen, z.B. Vögeln und Saccharopolyspora rectivirgula, geben IgA-Antikörper zusätzliche diagnostische Hinweise [16]. Sensitive Techniken wie der ELISA oder IFT sind vorzuziehen; mit diesen sind IgG-Antikörper in 80-95\% nachweisbar (in der Regel in hoher Konzentration), bei antigenexponierten Asymptomatikern, aber auch in $20-50 \%$ (meist in niedriger Konzentration) sowie bei Gesunden ohne bekannte Exposition in 1-8\% (in nied-
Tab. 2 Die häufigsten Antigene und Krankheitsbilder der EAA

\begin{tabular}{|lll|}
\hline Antigenquelle & Antigen & Krankheitsbild \\
\hline Vogelstaub & Vogelproteine & Vogelhalterlunge \\
\hline Bettfedern & Federnpuder & Bettfedern-Alveolitis \\
\hline $\begin{array}{l}\text { Heu- und } \\
\text { Strohstaub }\end{array}$ & $\begin{array}{l}\text { thermophile Aktinomy- } \\
\text { zeten und Schimmelpilze }\end{array}$ & Farmerlunge \\
\hline $\begin{array}{l}\text { Befeuchter- } \\
\text { wasser }\end{array}$ & $\begin{array}{l}\text { Bakterien, Schimmel- } \\
\text { pilze, Parasiten }\end{array}$ & Befeuchterlunge \\
\hline Holzstäube & Hölzer, Schimmelpilze & Holzarbeiterlunge \\
\hline $\begin{array}{l}\text { Schimmel } \\
\text { im Haus }\end{array}$ & Schimmelpilze & Innenraum-Alveolitis \\
\hline $\begin{array}{l}\text { Chemische } \\
\text { Substanzen }\end{array}$ & $\begin{array}{l}\text { Isozyanate, Anhydride } \\
\text { u.a. }\end{array}$ & Chemie-Alveolitis \\
\hline
\end{tabular}

riger Konzentration) [43]. Bei längerer Antigenkarenz (ab 6-12 Monaten) ist häufig ein Abfall der Antikörperkonzentration zu beobachten, nicht selten bis unter die Nachweisgrenze. Bei der Wellensittichhalterlunge etwa verschwinden die Antikörper während Antigenkarenz in 52\% der Fälle, davon 80\% innerhalb der ersten 2 Jahre [22]. Selten kommen auch während Antigenexposition keine Antikörper vor (seronegative EAA). Umgekehrt kommen Antikörper auch bei gesunden Antigen exponierten Personen vor, meist nur in niedriger Konzentration $[5,12,47]$.

\section{Lungenfunktion}

Bei der EAA können alle Funktionsbereiche (Ventilation, Diffusion und Distribution) betroffen sein. Charakteristisch sind eine restriktive Lungenfunktionsstörung mit Verminderung aller statischen Lungenvolumina sowie eine Gasaustauschstörung. Diese zeigt sich als eingeschränkte Diffusionskapazität bzw. in der Blutgasanalyse als Ruhe- und/oder Belastungshypoxämie. Noch sensitiver kann die arterio-alveoläre Sauerstoffdifferenz $\left(\mathrm{AaDO}_{2}\right)$ mittels Ergospirometrie eine Gasaustauschstörung anzeigen [40].

Bei ca. 25\% der Erkrankten liegt zusätzlich eine Bronchialobstruktion vor. Im Spätstadium der EAA wird auch eine Lungenüberblähung infolge eines Emphysems beobachtet $[8,19,44]$. In schätzungsweise $20-40 \%$ ist eine bronchiale Hyperreagibilität nachweisbar.

Die Lungenfunktionseinschränkungen können erheblich sein, selbst wenn die Symptome und das Röntgenbild nur geringe Störungen vermuten lassen und umgekehrt.

\section{Radiologie}

Im konventionellen Röntgen-Thoraxbild bei einer akuten EAA finden sich am häufigsten bilaterale milchglasartige Trübungen der mittleren und basalen Lungenabschnitte. Dies kann einem Lungenödem oder einem miliaren Muster ähneln. Seltener findet man massive Trübungsbezirke, Linien- und Ringschatten, verdickte Septallinien, Pleuraverdickung und kleine Mediastinallymphome $[8,24]$. Die Veränderungen der akuten EAA sind meist rasch reversibel. Bei der akuten und subakuten EAA kann das konventionelle Röntgenbild des Thorax in bis zu 30\% der Fälle unauffällig sein [34].

Bei der chronischen Verlaufsform stehen zunehmende retikulonoduläre Infiltrationen mit Betonung der mittleren und apikalen Lungenabschnitte im Vordergrund. Weiter können bei der chro- 
nischen EAA radiologische Zeichen des Emphysems und Cor pulmonale hinzukommen $[1,8]$.

Die Sensitivität der Computertomographie (CT) und insbesondere der hochauflösenden Computertomographie (HR-CT) des Thorax ist deutlich höher, ein negatives HR-CT schließt die EAA jedoch nicht aus [43].

Ein typischer Befund der akuten EAA im HR-CT ist eine diffuse milchglasartige Trübung aller Lungenabschnitte [8].

Bei der subakuten Verlaufsform finden sich charakteristische zentrilobuläre mikronoduläre, meist unscharf begrenzte Infiltrate von milchglasartiger Dichte und infolge eines Airtrapping ein mosaikartiges Muster. Ebenfalls kann ein tree-in-bud-Phänomen („Zweig mit Knospen“) aufgrund einer Broncholitis nachzuweisen sein [15].

Bei der chronischen EAA finden sich bevorzugt in den mittleren und apikalen Lungenabschnitten irreguläre lineare (retikuläre) Verdichtungsbezirke, teils auch Traktionsbronchiektasen und Honigwabenmuster [46]. Areale mit Milchglastrübung und zentrilobuläre noduläre Infiltrate können auch bei der chronischen EAA zu finden sein, stehen aber nicht im Vordergrund [32]. Der für die idiopathische Lungenfibrose charakteristische subpleurale Befall fehlt fast immer [23].

\section{Bronchoalveoläre Lavage}

Von allen interstitiellen Lungenkrankheiten weist die EAA die höchste Gesamtzellzahl auf. In der Regel besteht eine Lymphozytose (Normbereich < 15\%) [49]. Allerdings können antigenexponierte, nicht erkrankte Personen ebenfalls eine Lymphozytose in der bronchoalveolären Lavage (BAL) aufweisen, in der Regel jedoch weniger ausgeprägt (so genannte subklinische EAA). Dies gestattet nicht die Prognose, dass solche gesunden Personen später an einer EAA erkranken werden [9].

Der CD4/CD8-Quotient ist in den meisten Fällen von EAA erniedrigt $(<1,3)$, ein normaler oder erhöhter Quotient schließt eine EAA aber nicht aus $[10,11,33]$. Weitere BAL-Merkmale sind vermehrte Mastzellen, Plasmazellen und schaumige Makrophagen $[10,11,42]$.

Die bronchoalveoläre Lavage sollte frühestens 48 Stunden nach Antigenexposition durchgeführt werden, da in den ersten Stunden nach Antigenkontakt die Neutrophilen erhöht sind [13].

\section{Histologie}

Das histologische Bild der EAA ist durch eine vorwiegend intramurale, oft azinuszentral akzentuierte lymphozytäre Infiltration gekennzeichnet. In gut der Hälfte der Fälle sind zusätzlich teils uncharakteristische, teils auch epitheloidzellige, unregelmäßig im Parenchym verteilte Granulome sowie eine BOOP-Reaktion (Masson-Körperchen, Buds) nachweisbar [2]. Die Trias aus lymphozytärer intramuraler Alveolitis in Kombination mit Granulomen und BOOP-Reaktion entspricht dem Vollbild einer EAA und erlaubt die histologische Diagnose.

In fortgeschrittenen Fällen, bei chronischer Verlaufsform, kann sich eine interstitielle Fibrose entwickeln, so dass dann von histologischer Seite eine UIP und die fibrotische Variante der NSIP mit in die Differenzialdiagnose einbezogen werden müssen $[7,30]$. Die zelluläre Variante einer NSIP kann das einzige histologische Muster einer EAA darstellen, so dass vor der Diagnose- stellung einer idiopathischen NSIP immer eine EAA ausgeschlossen werden sollte [48].

Stehen epitheloidzellige Granulome im Vordergrund, muss an eine Sarkoidose gedacht werden [14].

Die BOOP-Reaktion bei EAA muss histologisch von einer kryptogen organisierenden Pneumonie (COP) als eigenständiges Krankheitsbild abgegrenzt werden [2].

Wenn die Untersuchung von repräsentativen bronchoskopischen Lungenbiopsien keine differenzialdiagnostische Klärung gestattet, was häufig der Fall ist [20], kann die Entnahme von größeren Gewebsproben durch eine offene Lungenbiopsie (z.B. VATS) erforderlich sein [30].

Wird histologisch eine unspezifische interstitielle Pneumonie ohne Fibrose (NSIP) oder eine BOOP-ähnliche Histologie nachgewiesen, so liegt klinisch meist eine akute Verlaufsform vor, während bei der chronischen Verlaufsform mit schlechterer Prognose häufiger eine UIP oder eine NSIP mit Fibrose gefunden wird [30].

\section{Karenztest}

Zahlreiche Antigene wie Bettfedern, Ziervögel, Zimmerspringbrunnen oder Arzneimittel lassen sich problemlos meiden, um eine Besserung der Krankheitssymptome zu erzielen. Optimal ist, die pathologisch veränderten klinischen Parameter, die Lungenfunktion, Leukozyten, CRP, Blutsenkung und andere Parameter unter der Karenz zeitlich zu verfolgen [43]. Bei Antigenen wie Schimmel in Räumen lässt sich Karenz nur durch ein vorübergehendes Verlassen des Arbeitsplatzes oder der Wohnung erreichen. Besonders aussagekräftig ist der Karenztest, wenn die abgeschwächten oder bereits ganz verschwundenen Symptome bei erneuter Exposition, z. B. nach einem Urlaub, wieder auftreten (Karenz-Reexpositionstest).

\section{Inhalativer Expositions- oder Provokationstest}

Eine inhalative Provokationstestung kann als Reexposition in der realen Umwelt (z.B. am Arbeitsplatz), als realitätsähnliche Provokation in der Klinik oder durch Inhalation von Extrakten der verdächtigten Substanzen durchgeführt werden. Die diagnostische Inhalation von Antigenen im Sinne einer realitätsähnlichen Provokation in der Klinik oder durch Inhalation von Extrakten der verdächtigten Substanzen sollte in der Regel nur stationär in speziellen Zentren mit Erfahrung mit inhalativen Provokationstestungen und der Möglichkeit einer Intensivtherapie durchgeführt werden, da schwerwiegende Hypoxämien auftreten können [27]. Der Test gilt als diagnostisch besonders zuverlässig [12]. Kontraindikationen einer Antigenexposition oder -provokation sind ein $\mathrm{pO}_{2}$ unter $60 \mathrm{~mm} \mathrm{Hg}$, eine VK und TK unter $50 \%$ des Sollwertes, ein akuter Infekt oder schwere Begleiterkrankung sowie eine Bronchialobstruktion mit einer Resistance von $\geq 0,5 \mathrm{kPa} \cdot \mathrm{s} / \mathrm{l}$ oder einer spezifischen Resistance von $\geq 2,0 \mathrm{kPa} \cdot \mathrm{s}$. Eine relative Kontraindikation ist die Behandlung mit Entzündungshemmern, insbesondere mit systemischen Kortikosteroiden. Weitere Informationen zu dem Test siehe „Empfehlungen zur inhalativen Provokationstestung bei exogen-allergischer Alveolitis“ der „Arbeitsgemeinschaft Exogen-allergische Alveolitis“ unter der Federführung von Müller-Wening [4]. 
Tab. 3 Diagnosekriterien der EAA

1. Antigen-Exposition
2. Expositions- und/oder zeitabhängige Symptome
3. Spezifische IgG-Antikörper im Serum
4. Sklerophonie (Knisterrasseln)
5. Röntgenzeichen der EAA, ggf. im HR-CT
6. $\mathrm{pO}_{2}$ in Ruhe und/oder bei Belastung erniedrigt oder DCO einge-
schränkt
Sind alle 6 Kriterien erfüllt, liegt eine EAA vor.
Fehlt eines der oben genannten Kriterien, so kann dieses durch ei-
nes der folgenden ersetzt werden:
Lymphozytose in der BAL
Mit EAA zu vereinbarender histopathologischer Befund der Lunge
Positiver Karenztest
Positive inhalative Expositions- oder Provokationstestung
Sind insgesamt 6 Kriterien erfüllt, liegt eine EAA vor.

\section{Diagnosestellung}

Die vier aussagekräftigsten nichtinvasiven Kriterien der EAA sind nach Lacasse u. Mitarb. [21]:

Nachweis einer Antigen-Exposition

Expositions-abhängige klinische Symptome 3-12 Stunden nach Antigenkontakt

Spezifische IgG-Antikörper im Serum

Sklerophonie (Knisterrasseln)

Weitere wichtige Diagnosekriterien sind:

EAA-typische Röntgenzeichen im konventionellen ThoraxRöntgenbild oder im HR-CT [15]

Arterielle Hypoxämie in Ruhe und/oder unter Belastung oder eine eingeschränkte Diffusionskapazität (DCO) [40].

Wir betrachten im Sinne einer rationellen Diagnostik die Diagnose einer exogen-allergischen Alveolitis als gesichert, wenn alle 6 Kriterien erfüllt sind.
Sind nicht alle 6 Diagnosekriterien erfüllt, so kann jedes der folgenden Kriterien jeweils ein fehlendes ersetzen:

Lymphozytose in der BAL

Ein mit einer EAA zu vereinbarender histopathologischer Befund der Lunge

Ein positiver Karenztest

Ein positiver inhalativer Expositions- oder Provokationstest

In diskrepanten Situationen kann der inhalative Expositionsoder Provokationstest den Ausschlag geben. Dies ist vor allem bei fehlendem Nachweis der Antigenquelle bzw. fehlenden spezifischen IgG-Antikörpern hilfreich.

In Tab. 3 sind diese neuen Diagnosekriterien übersichtlich zusammengefasst.

\section{Differenzialdiagnosen}

Eine wichtige Differenzialdiagnose ist die toxische Alveolitis, insbesondere das Organic Dust Toxic Syndrome (ODTS) (z.B. Drescherfieber, Befeuchterfieber), bei dem das Röntgenbild in der Regel normal ist, keine Lungenfunktions-(Diffusions-)störung der Lunge vorliegt und Antikörper in hoher Konzentration fehlen, in niedriger Konzentration kommen sie aber wie bei Gesunden in 1-8\% vor, bei bekannter Antigen-Exposition noch häufiger (bis zu 50\%) [43] ( Tab.4). Weitere Differenzialdiagnosen sind rezidivierende virale oder bakterielle Atemwegsinfekte, Pneumonien, Bronchitis, allergische und immunologische Lungenerkrankungen wie die bronchopulmonalen Mykosen, die Berylliose und Autoimmunerkrankungen, interstitielle Lungenerkrankungen, insbesondere die Pneumokoniosen, die Sarkoidose und die idiopathischen interstitiellen Pneumonien [43].

Tab. 4 Differenzialdiagnose ODTS - EAA (modifiziert nach Nowak 2006) [29]

\begin{tabular}{|c|c|c|}
\hline & Organic Dust Toxic Syndrome & Exogen-allergische Alveolitis \\
\hline $\begin{array}{l}\text { mehrere exponierte Personen befallen } \\
\text { (Cluster) }\end{array}$ & ja & ungewöhnlich \\
\hline Raucheranamnese & Nichtraucher überwiegen & Nichtraucher überwiegen \\
\hline Expositionsanamnese & $\begin{array}{l}\text { organische Aerosole, schimmeliges Getreide, Silage, Heu, } \\
\text { Holzhackschnitzel, Dämpfe von kontaminiertem Wasser, } \\
\text { Exkremente von Tieren } \\
\text { Symptomatik kann nach erstmaliger Exposition auftreten }\end{array}$ & $\begin{array}{l}\text { wiederholte (!) Exposition gegenüber } \\
\text { Antigenen }\end{array}$ \\
\hline Auslöser & Endotoxine, Glucane Mykotoxine, andere? & Die Antigene der EAA \\
\hline Latenzzeit & $4-12$ Stunden & $4-12$ Stunden \\
\hline Dauer eines Schubes & 1 Tag, höchstens 2 - 3 Tage & mehrere Tage bis Wochen \\
\hline Symptomatik & $\begin{array}{l}\text { Husten, Frösteln, Fieber, Abgeschlagenheit, Myalgien, } \\
\text { Kopfschmerzen }\end{array}$ & $\begin{array}{l}\text { Fieber, Frösteln, Abgeschlagenheit, Husten, } \\
\text { Kurzluftigkeit }\end{array}$ \\
\hline körperlicher Untersuchungsbefund & normal oder vereinzelte Rasselgeräusche & endinspiratorisches Knisterrasseln \\
\hline Röntgen Lunge & normal, allenfalls diskrete Infiltrate am 1 . Tag & häufig pathologische Lungenveränderungen \\
\hline Blutgasanalyse & normal, selten geringe Hypoxämie & Hypoxämie \\
\hline Lungenfunktion & normal, selten akut leicht restriktiv & Restriktion, Diffusionsstörung \\
\hline Typ III-Antikörper & meist negativ & meist positiv \\
\hline bronchoalveoläre Lavage & Neutrophilie & $\begin{array}{l}\text { Obligat Lymphozytose, häufig zusätzlich } \\
\text { CD4/CD8-Quotient erniedrigt, in der akuten } \\
\text { Phase zusätzlich Neutrophilie }\end{array}$ \\
\hline Prognose & gut, Tendenz zur COPD & variabel, Tendenz zur Lungenfibrose \\
\hline Schleimhäute & gerötet & normal \\
\hline Inzidenz (pro 10000 und Jahr) & 20 - 190 (Drescherfieber) & $2-30$ (Farmerlunge) \\
\hline
\end{tabular}




\section{Anmerkung}

Abdruck mit freundlicher Genehmigung des Dustri Verlags Dr. Karl Feistle aus „Allergologie 2006; 29: 431 - 438

\section{Institutsangaben}

Labor und Praxis Prof. Sennekamp, Bonn

Zusamklinik Deutsche Rentenversicherung Schwaben, Zusmarshausen

Institut für Pathologie, Diakoniekrankenhaus Rotenburg/Wümme

Ordinariat und Zentralinstitut für Arbeitsmedizin der Universität Hamburg

Charité Berlin

Ruhrlandklinik Essen

Krankenhaus Großhansdorf

Fachkrankenhaus Coswig

ehemals Herz-Lungenpraxis Stade

${ }^{10}$ Lungenklinik Lostau

11 Institut und Poliklinik für Arbeits- und Umweltmedizin der Universität München

12 Städtisches Klinikum Dessau

13 Universitätsklinik für Innere Medizin Marburg

\section{Literatur}

1 Adler BD, Padley SPG, Müller NL et al. Chronic hypersensitivity pneumonitis: high-resolution CT and radiographic features in 16 patients. Radiology 1992; 185: 91 - 95

2 Amthor MJ. Histologische Differenzialdiagnose der exogen-allergischen Alveolitis EAA unter besonderer Berücksichtigung der Bronchiolitis obliterans mit organisierender Pneumonie (BOOP). Allergologie 2003; 26: $310-312$

3 Arbeitsgemeinschaft exogen-allergische Alveolitis. Empfehlungen zur Diagnosestellung einer exogen-allergischen Alveolitis. Allergologie 1990; 13: 111 - 112

4 Arbeitsgemeinschaft exogen-allergische Alveolitis. Empfehlungen zur inhalativen Provokationstestung bei exogen-allergischer Alveolitis. Pneumologie 1998; 52: 444-446

5 Baur X, Richter G, Pethran A et al. Increased prevalence of IgG-induced sensitization and hypersensitivity pneumonitis (humidifier) lung in nonsmokers exposed to aerosols of a contaminated air conditioner. Respiration 1992; 59: $211-214$

6 Bergmann K-Ch, Branski H, Götz M et al. Empfehlungen zur Definition des Krankheitsbildes allergische Alveolitis. Z Erkrank Atm-Org 1978; 151: $167-171$

7 Churg A, Muller NL, Flint J et al. Chronic hypersensitivity pneumonitis. Am J Surg Pathol 2006; 30: 2001 - 208

8 Cormier Y, Brown M, Worthy $S$ et al. High resolution computed tomographic characteristics in acute farmer's lung and its follow up. Eur Respir J 2000; 16: 56-60

9 Cormier Y, Létourneau L, Racine G. Significance of precipitins and asymptomatic lymphocytic alveolitis: a 20-yr follow-up. Eur Respir J 2004; 23: $523-525$

10 Costabel U. Atlas der bronchoalveolären Lavage. Stuttgart: Thieme, 1994

11 Costabel U, Guzman J. Bronchoalveolar lavage. In: Schwarz MI, King TE $\mathrm{Jr}$ (eds). Interstitial lung disease. Fourth edition. London, Hamilton: BC Decker, 2003: 452 - 484

12 Fink JN, Ortega HG, Reynolds HY et al. Needs and opportunities for research in hypersensitivity pneumonitis. Am J Respir Crit Care Med 2005; 171: $792-798$

13 Fournier E, Tonnel AB, Gosset $P$ et al. Early neutrophil alveolitis after antigen inhalation in hypersensitivity pneumonitis. Chest $1985 ; 88$ : $563-566$

14 Gal AA, Koss MN. Pulmonary disorders. In: Epstein JI (eds). Differential diagnosis in pathology. Baltimore: Williams and Wilkins, 1997: 26-27

15 Hartman TE. The HRCT features of extrinsic allergic alveolitis. Semin Respir Crit Care Med 2003; 24: 419-426

16 Huizinga $M$, Berrens $L$. Detection of class-specific antibodies against Micropolyspora faeni antigens in farmer's lung. Clin Allergy 1985; 15: $139-145$

17 Koschel D, Stark W, Karmann F et al. Extrinsic allergic alveolitis caused by misting fountains. Resp Med 2005; 99: 943-947

18 Koschel D, Pietrzyk C, Sennekamp J et al. Schwimmbadlunge - Exogenallergische Alveolitis oder Mykobakteriose? Pneumologie 2006; 60: $285-289$

19 Kroidl RF. Lungenemphysem, Endstadium der allergischen Alveolitis. Allergologie 1994; 17: 74-75
20 Lacasse Y, Fraser RS, Fournier M et al. Diagnostic accuracy of transbronchial biopsy in acute farmer's lung disease. Chest 1997; 112: 14591465

21 Lacasse $Y$, Selman $M$, Costabel $U$ et al. Clinical diagnosis of hypersensitivity pneumonitis. Am J Respir Crit Care Med 2003; 168: 952 - 958

22 Lee TH, Wraith DG, Bennett CO et al. Budgerigar fancier's lung. Clin Allergy 1983; 13: $197-202$

23 Lynch DA, Newell JD, Logan PM et al. Can CT distinguish hypersensitivity pneumonitis from idiopathic pulmonary fibrosis? Amer J Radiol 1995; 165: $807-811$

24 Matar LD, McAdams HP, Sporn TA. Hypersensitivity pneumonitis. Am J Roentgenol 2000; 174: 1061 - 1066

25 Mohr LC. Hypersensitivity pneumonitis. Curr Opin Pulm Med 2004; 10: $401-411$

26 Morris DG. Gold, silver, and bronze. Am J Respir Crit Care Med 2003; 168: 909 - 917

27 Müller-Wening D. Erfahrungen zur inhalativen Allergenprovokation bei exogen-allergischer Alveolitis. Allergologie 1992; 15: 2-14

28 Müller-Wening $D$, Koschel $D$, Stark $W$ et al. Befeuchterassoziierte Erkrankungen bei der Allgemeinbevölkerung. Dtsch Med Wschr 2006; 131: 491 - 496

29 Nowak D. Arbeitsmedizin. München: Urban \& Fischer, 2006

30 Ohtani Y, Saiki S, Kitaichi M et al. Chronic bird fancier's lung: histopathological and clinical correlation. Thorax 2005; 60: 665-671

31 Patel AM, Ryu JH, Reed CE. Hypersensitivity pneumonitis: current concepts and future questions. J Allergy Clin Immunol 2001; 108: 661 670

32 Raoof S, Amchentsev A, Vlahos I et al. Pictorial essay: multinodular disease: a high-resolution CT scan diagnostic algorithm. Chest 2006; 129: $805-815$

33 Ratjen $F$, Costabel $U$, Griese $M$ et al. Bronchoalveolar lavage fluid findings in children with hypersensitivity pneumonitis. Eur Respir J 2003; 21: $144-148$

34 Remy-Jardin M, Remy J, Wallaert B et al. Subacute and chronic bird breeder hypersensitivity pneumonitis: sequential evaluation with CT and correlation with lung function tests and bronchoalveolar lavage. Radiology 1993; 189: 111 - 118

35 Richerson HB, Bernstein IL, Fink JN et al. Guidelines for the clinical evaluation of hypersensitivity pneumonitis. J Allergy Clin Immunol 1989; 84: $839-844$

36 Rickman OB, Ryu JH, Fidler ME et al. Hypersensitivity pneumonitis associated with Mycobacterium avium complex and hot tub use. Mayo Clin Proc 2002; 77: 1233-1237

37 Rolke M, Engelhart S, Kämpfer P et al. Exogen-allergische Alveolitis von einem Dampfbügeleisen. Allergologie 2003; 26: 327 - 328

38 Schreiber J, Knolle J, Heerdt $S$ et al. Primär chronischer Verlauf einer exogen-allergischen Alveolitis durch Diisozyanate bei einer Sekretärin infolge indirekter Exposition. Atemw-Lungenkrkh 2006; 32: 160 166

39 Schuyler $M$, Cormier $Y$. The diagnosis of hypersensitivity pneumonitis. Chest 1997; 111: $534-536$

40 Schwaiblmair M, Beinert T, Vogelmeier $C$ et al. Cardiopulmonary exercise testing following hay exposure challenge in farmer's lung. Eur Respir J 1997; 10: 2360 - 2365

41 Selman $M$. Hypersensitivity pneumonitis: a multifaceted deceiving disorder. Clin Chest Med 2004; 25: 531 - 547

42 Semenzato G, Bjermer L, Costabel $U$ et al. Clinical role of bronchoalveolar lavage in extrinsic allergic alveolitis. Eur Respir Rev 1992; 8: 6974

43 Sennekamp J. Extrinsic allergic alveolitis - hypersensitivity pneumonitis. München: Dustri, 2004

44 Sovijärvi AR, Kuusisto P, Muittari A et al. Trapped air in extrinsic allergic alveolitis. Respiration 1980; 40: $57-64$

45 Terho EO. Diagnostic criteria for farmer's lung disease. Amer J Industr Med 1986; 10: 329

46 Tuengerthal S. HRCT der exogen-allergischen Alveolitis. Allergologie 2001; 24: $136-137$

47 Vogelmeier C, Mazur G, Pethran A et al. Immunpathogenese der exogen-allergischen Alveolitis. Immun Infekt 1995; 23: 86-91

48 Vourlekis JS, Schwarz MI, Cool CD et al. Nonspecific interstitial pneumonitis as the sole histologic expression of hypersensitivity pneumonitis. Amer J Med 2002; 112: 490 - 493

49 Welker L, Jörres RA, Costabel U et al. Predictive value of BAL cell differentials in the diagnosis of interstitial lung diseases. Eur Respir J 2004; 24 : $1000-1006$ 